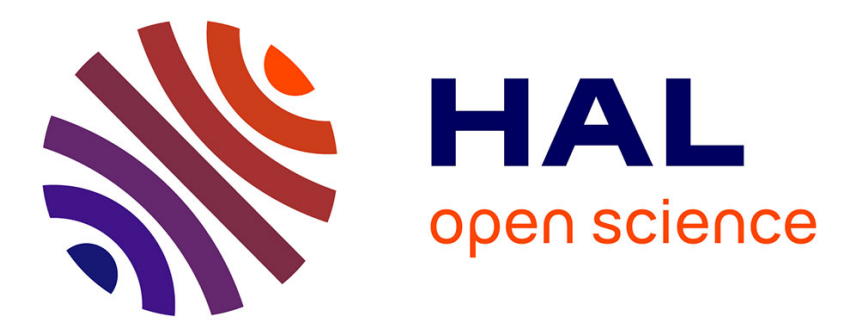

\title{
Control of PEMFC Air-Feed System using Lyapunov-based Robust and Adaptive Higher Order Sliding ModeControl
}

\author{
Salah Laghrouche, M. Harmouche, F.S. Ahmed, Yacine Chitour
}

\section{To cite this version:}

Salah Laghrouche, M. Harmouche, F.S. Ahmed, Yacine Chitour. Control of PEMFC Air-Feed System using Lyapunov-based Robust and Adaptive Higher Order Sliding ModeControl. IEEE Transactions on Control Systems Technology, 2015, 23 (4), pp.1594-1601. 10.1109/tcst.2014.2371826 . hal-01271294

HAL Id: hal-01271294

https://hal-centralesupelec.archives-ouvertes.fr/hal-01271294

Submitted on 3 Apr 2020

HAL is a multi-disciplinary open access archive for the deposit and dissemination of scientific research documents, whether they are published or not. The documents may come from teaching and research institutions in France or abroad, or from public or private research centers.
L'archive ouverte pluridisciplinaire HAL, est destinée au dépôt et à la diffusion de documents scientifiques de niveau recherche, publiés ou non, émanant des établissements d'enseignement et de recherche français ou étrangers, des laboratoires publics ou privés. 


\title{
Control of PEMFC Air-Feed System Using Lyapunov-Based Robust and Adaptive Higher Order Sliding Mode Control
}

\author{
Salah Laghrouche, Mohamed Harmouche, Fayez Shakil Ahmed, and Yacine Chitour
}

\begin{abstract}
In this brief, we present Lyapunov-based robust and adaptive higher order sliding mode (HOSM) controllers for the air-feed system of polymer electrolyte membrane fuel cells, which is a nonlinear single-input, single-output system with bounded uncertainty. The system consists of a motorized compressor, which is driven at its optimal point in order to minimize the internal energy consumption of the system. This brief provides an experimental demonstration of the applicability of the recently developed fixed-gain robust controller and adaptive controller for this problem. Third-order controllers are developed in order to obtain a continuous profile for the input current of the compressor motor. In this regard, a complete adaptive arbitrary HOSM control has been presented for the first time, with Lyapunov-based proof. A performance comparison between the two controllers is presented in the end.
\end{abstract}

Index Terms-Adaptive control, finite time stabilization, higher order sliding mode (HOSM), Lyapunov analysis, polymer electrolyte membrane fuel cell (PEMFC), robust control.

\section{INTRODUCTION}

$\mathbf{F}$ UEL cells and their auxiliary systems pose challenging control problems, as they are typically nonlinear and difficult to characterize. They require robust or adaptive control methods, as their physical parameters are uncertain, varying with operating conditions and environmental effects. One important control problem in polymer electrolyte membrane fuel cell (PEMFC) systems is the minimization of the power consumed internally by their air-feed systems, in order to maximize the net power output. In particular, a PEMFC needs a sufficient quantity of excess air (oxygen excess) in its cathode in order to respond to load variations and transitions without damaging itself [1]. On the other hand, it has been established that the power consumption of air-feed compressors is the highest among all auxiliary systems of the fuel cell, rising up to $20 \%$ of the total PEMFC

Manuscript received January 15, 2014; revised July 7, 2014; accepted October 8, 2014. Manuscript received in final form November 13, 2014. This work was supported by the iCODE Institute through the Research Project of Idex Paris-Saclay. Recommended by Associate Editor A. Zolotas.

S. Laghrouche is with the Laboratoire OPERA, Universite de Technologie de Belfort-Montbéliard, Belfort 90010, France (e-mail: salah.laghrouche@utbm.fr).

M. Harmouche is with Actility, Paris 75899, France (e-mail: mohamed.harmouche@actility.com).

F. S. Ahmed is with the Laboratoire d'Automatique et de Génie des Procédés, Université Claude Bernard Lyon 1, Villeurbanne F-69622, France (e-mail: fahmed@lagep.univ-lyon1.fr).

Y. Chitour is with the Laboratoire des Signaux et Systèmes, Université Paris XI, Gif-sur-Yvette 91192, France (e-mail: yacine.chitour@1ss.supelecc.fr).

Color versions of one or more of the figures in this paper are available online at http://ieeexplore.ieee.org.

Digital Object Identifier 10.1109/TCST.2014.2371826 power output [1]. Therefore, the air-feed system requires precise control for running the compressor at its optimal operating point, thereby maximizing the net power output of the fuel cell while keeping the oxygen excess ratio high enough for proper operation [2], [3].

Higher order sliding mode control (HOSMC) [4] is a wellestablished control strategy for uncertain nonlinear systems, as it is insensitive to parametric uncertainty and external disturbance. Unlike classical sliding mode, HOSMC does not suffer from high-frequency chattering because the characteristic discontinuous control [5] acts upon a higher derivative of the sliding variable. If the bounds of parametric uncertainty in the system are known, then fixed-gain HOSM controllers can be designed with relative ease. However, this is usually difficult in practical cases, as the estimation of uncertainty bounds requires rigorous experimentation in worst case conditions. In these cases, adaptive-gain (or simply adaptive) controllers provide a successful means of controlling the system through dynamically adapting gains. However, these controllers ensure practical convergence only, i.e., to a neighborhood of the origin. Many fixed-gain arbitrary robust HOSMC algorithms exist in contemporary literature, prominent examples being [6]-[9]. Huang et al. [10] were the first to use dynamic gain adaptation in SMC for the problem of unknown uncertainty bound, following [11] and [12]. Other works in this domain include [13]-[16]. These contributions remain limited to first- and second-order sliding mode. Initial findings on Lyapunov-based robust and (partial) adaptive arbitrary HOSMC were recently presented in [17].

Sliding mode controllers have been studied for PEMFC air-feed system control as well in [18]-[20], and two important examples of second-order SMC (SOSMC) are [21] and [22]. In the former, the oxygen excess ratio is assumed to have a static relationship with the compressor flow rate, and the compressor is controlled using SOSMC. In the latter, the authors have proposed a dual loop Cascade SOSMC controller, which address the compressor speed reference and current control individually. This approach is more practical as both the control loops are robust; however, its implementation requires different loop rates for controllers. Approaching this problem by third-order-sliding-mode-based oxygen excess ratio controllers appears to be a better approach in comparison with both these methods, as third-order extension results in continuous current control of the motocompressor. Adaptive third-order controllers would provide further ease in control design as precise parameters of the otherwise complex system need not be known. 


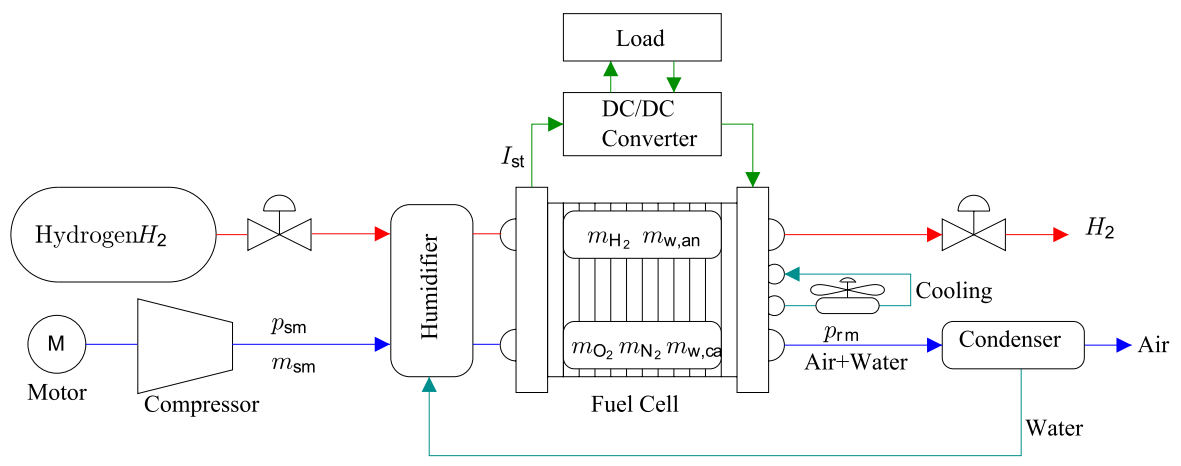

Fig. 1. Fuel cell air-feed system.

In this brief, the robust and adaptive approaches of [17] are extended and applied to the HOSM control of the PEMFC air-feed systems. The objective is to operate the air-feed compressor at its optimal point with respect to the load current, thereby maintaining the required oxygen excess ratio while keeping the power consumption low. There are two major contributions in this brief. First, it is shown that if the bounds of parametric uncertainty are known, then the air-feed control problem can be practically solved with the third-order robust HOSMC controller of [17]. The second main contribution in this brief is the extension of the partial adaptive controller presented in [17] to develop, for the first time, a complete adaptive arbitrary order sliding mode controller. The adaptation dynamics use a saturation function that results in rapid increase as well as rapid decrease of gains when the sliding variable and its derivatives are, respectively, outside and inside of a defined neighborhood of zero. The advantages of this adaptive controller design are its arbitrary order and its fast adaptation rates in both directions. The Lyapunov function defines sufficient conditions for controller parameters in order to ensure convergence to a defined neighborhood. The practical applicability of both the robust and adaptive controllers is demonstrated on a hardware-in-loop (HIL) airfeed test bench, using a real-time physical PEMFC emulator. A comparative analysis of the performances of the robust and adaptive controller is also presented.

This brief is organized as follows. The PEMFC air-feed system is described in Section II. HOSM problem formulation and robust and adaptive control design are discussed in Section III. Their implementation and experimental results are shown in Section IV. Finally, the conclusions are drawn in Section V.

\section{PEMFC AIR-FEED SySteM}

The PEMFC air-feed system (Fig. 1) feeds the fuel cell cathode with air (as the source of oxygen). It consists of a motocompressor and a manifold, in which a certain quantity of air (oxygen excess) is maintained such that the reaction between hydrogen and oxygen is continuous, without any interruption. Maintaining sufficient oxygen excess is critical for the PEMFC, as insufficient air leads to oxygen starvation during load transitions, where a sudden high load imposes a sudden increase in the rate of reaction. The dynamic model of a fuel cell air-feed system is as follows [22]:

$$
\begin{aligned}
\dot{x}_{1}= & c_{1}\left(x_{4}-x_{1}-x_{2}-c_{2}\right) \\
& -\frac{c_{3} x_{1}}{c_{4} x_{1}+c_{5} x_{2}+c_{6}} c_{17} \sqrt{x_{1}+x_{2}+c_{2}-c_{11}}-c_{7} \zeta \\
\dot{x}_{2}= & c_{8}\left(x_{4}-x_{1}-x_{2}-c_{2}\right) \\
& -\frac{c_{3} x_{2}}{c_{4} x_{1}+c_{5} x_{2}+c_{6}} c_{17} \sqrt{x_{1}+x_{2}+c_{2}-c_{11}} \\
\dot{x}_{3}= & -c_{9} x_{3}-c_{10}\left[\left(\frac{x_{4}}{c_{11}}\right)^{c_{12}}-1\right]+c_{13} u \\
\dot{x}_{4}= & c_{14}\left[1+c_{15}\left[\left(\frac{x_{4}}{c_{11}}\right)^{c_{12}}-1\right]\right] \\
& {\left[W_{\mathrm{cp}}-c_{16}\left(x_{4}-x_{1}-x_{2}-c_{2}\right)\right] } \\
u= & I_{q}, \quad \zeta=I_{\mathrm{st}}, \quad W_{\mathrm{cp}}=c_{21} \omega_{\mathrm{cp}} .
\end{aligned}
$$

The physical quantities that form the state vector $x$ are

$$
x=\left[\begin{array}{llll}
x_{1} & x_{2} & x_{3} & x_{4}
\end{array}\right]^{T}=\left[\begin{array}{llll}
p_{\mathrm{O}_{2}} & p_{\mathrm{N}_{2}} & \omega_{\mathrm{cp}} & p_{\mathrm{sm}}
\end{array}\right]^{T}
$$

where $p_{\mathrm{O}_{2}}$ and $p_{\mathrm{N}_{2}}$ represent the oxygen partial pressure and the nitrogen partial pressure, respectively. The compressor speed is denoted by $\omega_{\mathrm{cp}}$ and the supply manifold pressure is denoted by $p_{\mathrm{sm}}$. The control input $u$ is the motor current, whereas the fuel cell stack current $\zeta$ is considered as measurable input disturbance. The compressor airflow is denoted by $W_{\mathrm{cp}}$ and it is proportional to the compressor speed. The parameters $c_{i}$ are considered as uncertain constants, decomposed as $c_{i}=c_{0 i}+\delta c_{i}$, where $c_{0 i}$ and $\delta c_{i}$ are the nominal value and the uncertainty of $c_{i}$, respectively. Complete details and physical significance of these parameters can be found in [22].

\section{A. Control Objective}

The control problem in the PEMFC system is to ensure a certain excess amount of air in the cathode while minimizing the energy consumed by the air-feed compressor. The oxygen excess ratio can be written as [22]

$$
\lambda_{\mathrm{O}_{2}}=\frac{c_{19}}{c_{20} \zeta}\left(x_{4}-x_{1}-x_{2}-c_{2}\right) .
$$

The net electrical power is optimized by reducing the consumption of the compressor, i.e., maintaining the oxygen excess ratio $\lambda_{\mathrm{O}_{2}}$ at its reference optimal value $\lambda_{\mathrm{O}_{2}}$,ref, which is determined as a function of the stack current $\zeta$ [22]

$$
\begin{aligned}
\lambda_{\mathrm{O}_{2}, \text { ref }}= & 5 \times 10^{-8} \zeta^{3}-2.87 \times 10^{-5} \zeta^{2}+2.23 \\
& \times 10^{-3} \zeta+2.5 .
\end{aligned}
$$


Our objective is to force $\lambda_{\mathrm{O}_{2}}$ to follow $\lambda_{\mathrm{O}_{2} \text {, ref }}$ in finite time.

\section{Higher Order SLiding Mode Controllers}

In this section, we will first recall the preliminary formulation of the HOSM control problem and the robust HOSM controller of [17]. Then, based on the initial findings of [17], the complete adaptive HOSM controller will be presented. Let us consider an uncertain nonlinear system

$$
\left\{\begin{array}{l}
\dot{x}(t)=f(x, t)+g(x, t) u \\
y(t)=s(x, t)
\end{array}\right.
$$

where $x \in \mathbb{R}^{n}$ is the state vector and $u \in \mathbb{R}$ is the control input. The sliding variable $s$ is a measured smooth outputfeedback function and $f(x, t)$ and $g(x, t)$ are uncertain smooth functions. It is assumed that the relative degree $r$ of the system is globally well defined, uniform, and time-invariant, and the associated zero dynamics are asymptotically stable [9]. Then, for suitable functions $\tilde{\varphi}(x, t)$ and $\tilde{\gamma}(x, t),(5)$ can be rewritten as

$$
y^{(r)}(t)=\tilde{\varphi}(x(t), t)+\tilde{\gamma}(x(t), t) u(t) .
$$

The functions $\tilde{\gamma}(x(t), t)$ and $\tilde{\varphi}(x(t), t)$ are assumed to be bounded by positive constants $\gamma_{m}, \gamma_{M}$, and $\bar{\varphi}$, such that

$$
0<\gamma_{m} \leq \tilde{\gamma}(x(t), t) \leq \gamma_{M}, \quad|\tilde{\varphi}(x(t), t)| \leq \bar{\varphi} .
$$

Defining $s^{(i)}:=d^{i} / d t^{i} y$, the goal of $r$ th order SMC is to arrive at, and keep the following manifold in finite time:

$$
s^{(0)}(x, t)=s^{(1)}(x, t)=\cdots=s^{(r-1)}(x, t)=0 .
$$

To be more precise, for $z=\left[\begin{array}{lll}z_{1} & z_{2} \ldots z_{r}\end{array}\right]^{T}:=\left[\begin{array}{l}s \dot{s} \ldots s^{(r-1)}\end{array}\right]^{T}$, (8) is equivalent to $z=0$. It is natural to replace (5) with a more general control system based on (7)

$$
\begin{aligned}
& \dot{z}_{i}=z_{i+1}, \quad i=1, \ldots, r-1 \\
& \dot{z}_{r}=\varphi(t)+\gamma(t) u \in I_{\varphi}+u I_{\gamma}
\end{aligned}
$$

where the new functions $\varphi$ and $\gamma$ are arbitrary measurable functions, bounded such that

$$
\varphi(t) \in[-\bar{\varphi}, \bar{\varphi}], \quad \gamma(t) \in\left[\gamma_{m}, \gamma_{M}\right]
$$

where $\bar{\varphi}, \gamma_{m}$, and $\gamma_{M}$ are positive constants. This system represents a perturbed integrator chain. The objective of this brief is to design controllers that stabilize (9) to the origin, ideally in finite time. Since these controllers are to be discontinuous feedback laws $u=U(z)$, solutions of (9) need to be understood here in Filippov sense [23].

\section{A. Robust Higher Order Sliding Mode Controller}

Let us first recall the robust controller [17], which is designed to (9), assuming that the bounds $\bar{\varphi}, \gamma_{m}$, and $\gamma_{M}$ are known. This controller has been derived from a class of Lyapunov-based controllers that guarantee finitetime stabilization of pure chain of integrators $(\varphi \equiv 0$ and $\gamma \equiv 1)$ [24], and satisfy certain additional geometric conditions. Let us consider that the initial states of the system are in a neighborhood of origin, $\hat{U} \subset \mathbb{R}^{r}$. Then, the main result of [17] is given as follows.
Theorem 1: If there exists a controller $u_{0}(z)$ that stabilizes a pure integrator chain in finite time and there exists a $C^{1}$ function $V_{1}$ defined on the neighborhood $\hat{U} \subset \mathbb{R}^{r}$, such that:

1) $\dot{V}_{1}+c V_{1}^{\alpha}(z(t)) \leqslant 0$, if $z(t) \in \hat{U}$;

2) $\frac{\partial V_{1}}{\partial z_{r}} u_{0} \leq 0$

3) $u_{0}=0 \Rightarrow \frac{\partial V_{1}}{\partial z_{r}}=0$.

Then, the following control law establishes HOSM on (9) with respect to $s$ :

$$
u=\frac{1}{\gamma_{m}}\left(u_{0}+\bar{\varphi} \operatorname{sign}\left(u_{0}\right)\right) .
$$

The detailed proof of this theorem can be found in [17]. It can be verified that many controllers, such as those of [25] and [26], fulfill the conditions demanded in Theorem 2. For the rest of this brief, we consider Hong's controller [25], which is defined as follows.

Let $k<0$ and $l_{1}, \ldots, l_{r}$ positive real numbers and $\lfloor a\rceil^{\theta}:=$ $|a|^{\theta} \operatorname{sign}(a), \forall a \in \mathbb{R}, \theta>0$. For $z=\left(z_{1}, \ldots, z_{r}\right)$, we define for $i=0, \ldots, r-1$

$$
\begin{aligned}
& p_{i}=1+(i-1) k \\
& v_{0}=0, \quad v_{i+1}=-l_{i+1}\left\lfloor\left\lfloor z_{i+1}\right\rceil^{\beta_{i}}-\left\lfloor v_{i}\right\rceil^{\beta_{i}}\right\rceil^{\left(\alpha_{i+1} /\left(\beta_{i}\right)\right.}
\end{aligned}
$$

where $\alpha_{i}=p_{i+1} / p_{i}$, for $i=1, \ldots, r$, and, for $k<0$ sufficiently small, we have $\beta_{0}=p_{2},\left(\beta_{i}+1\right) p_{i+1}=\beta_{0}+1>0$, $i=1, \ldots, r-1$.

\section{B. Adaptive Controller}

Let us now consider the case where uncertainty bounds $\gamma_{m}, \gamma_{M}$, and $\bar{\varphi}$ of (9) are unknown. In [17], this problem was partially solved and a controller was developed which could function without any explicit knowledge of $\bar{\varphi}$. We now present a complete arbitrary HOSM controller that can be designed without the knowledge of bounds of either uncertain function. Let us first define $\sigma(a)$ as the standard saturation function, $\sigma(a)=(a / \max (1,|a|)), a \in \mathbb{R}$. For $\varepsilon>0, a \in \mathbb{R}$, we define

$$
\nu_{\varepsilon}(a)=\frac{1}{2}+\frac{1}{2} \sigma\left(\frac{|a|-\frac{3}{4} \varepsilon}{\frac{1}{4} \varepsilon}\right) .
$$

We now propose the following controller:

$$
u=\hat{\gamma} u_{0}(z)+\hat{\varphi} \operatorname{sign}\left(u_{0}(z)\right)
$$

where $u_{0}$ is a homogeneous controller, as defined in Theorem 1. The adaptive function $\hat{\gamma}=\kappa+\delta\left|u_{0}(z)\right|$ and $\hat{\varphi}(t)$ is defined by the ordinary differential equation

$$
\dot{\hat{\varphi}}(t)=k v_{\varepsilon}\left(V_{1}(z)\right)-\left(1-v_{\varepsilon}\left(V_{1}(z)\right)\right)\lfloor\hat{\varphi}\rceil^{\eta}
$$

with the initial condition $\hat{\varphi}(0)=0$. The new terms are defined as $\kappa, \delta>0, \eta \in(0,1), k>0$, and $V_{1}$ is a homogeneous Lyapunov function which also satisfies Theorem 1 . The following theorem provides the main result for the adaptive controller.

Theorem 2: Consider (9) under the feedback control law (13). Then, $\forall \varepsilon, \exists c^{\prime}>0$ and $0<\alpha^{\prime}<1$ such that the following conditions are satisfied for any initial condition $z_{0} \in \hat{U}$. 


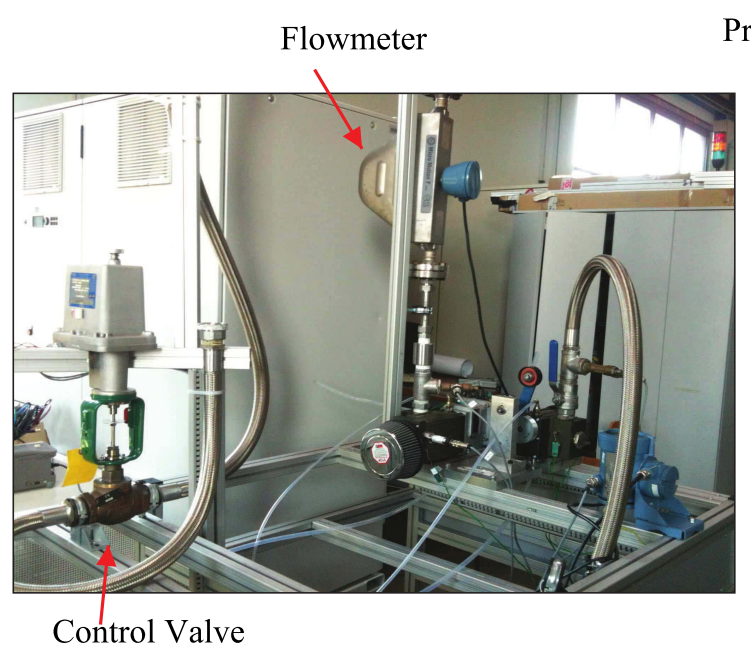

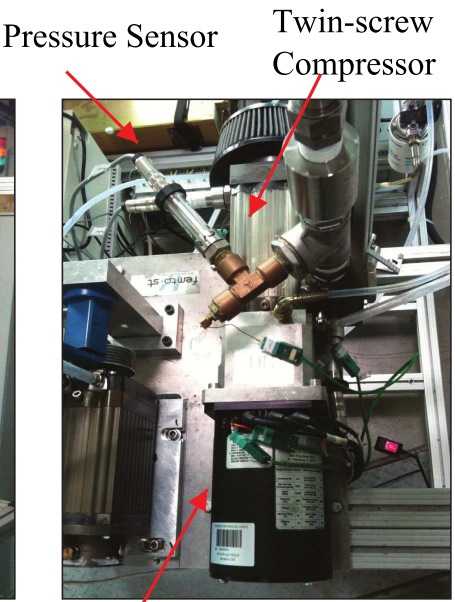

Motor

Fig. 2. Test bench.

$\mathrm{AQ}: 3250$
1) $\liminf _{t \rightarrow \infty} V_{1}(z(t)) \leq \varepsilon, \quad \lim \sup _{t \rightarrow \infty} V_{1}(z(t)) \leq \Delta$.

2) $\limsup _{t \rightarrow \infty}|\hat{\varphi}| \leq 2 \bar{\Phi}+k\left(\Delta^{1-\alpha} /(c(1-\alpha))\right)$

where

$$
\begin{aligned}
\bar{\Phi} & :=\frac{1}{\gamma_{m}}\left(\bar{\varphi}+\frac{\left(\kappa \gamma_{m}-1\right)^{2}}{4 \gamma_{m} \delta}\right) \\
\Delta & :=\left(\varepsilon^{1-\alpha^{\prime}}+\frac{c^{\prime}\left(1-\alpha^{\prime}\right) \gamma_{m}}{2 k} \bar{\Phi}^{2}\right)^{\frac{1}{1-\alpha^{\prime}}} .
\end{aligned}
$$

Proof of Theorem 2: We first demonstrate that the controller brings the system states from any domain $V_{1}>\varepsilon$ to the domain $V_{1} \leq \varepsilon$ in finite time. Then, it is proved that once $z$ reaches the domain $V_{1} \leq \varepsilon$, it stays in the domain $V_{1} \leq \Delta$ for all consecutive time instances and $\hat{\varphi}$ is upper bounded after a sufficiently large time.

Lemma 1 [17]: The function $\hat{\varphi}$ is non-negative and is defined as long as the trajectory of $z$ is defined.

Now, let us use contradiction again, to show $\liminf _{t \rightarrow \infty} V_{1}(z(t)) \leq \varepsilon$. Supposing that there exists $\bar{t}$ such that $V_{1}(t)>\varepsilon$ for every $t \geq \bar{t}$, then according to the dynamics of $\hat{\varphi}$, we get $\dot{\hat{\varphi}}=k$ for $t \geq \bar{t}$. This implies that for $t \geq \bar{t}, \hat{\varphi}$ is increasing and $\hat{\varphi}>\bar{\Phi}$. The derivative of the Lyapunov function is

$$
\begin{aligned}
\dot{V}_{1}= & \frac{\partial V_{1}}{\partial z_{1}} z_{2}+\cdots+\frac{\partial V_{1}}{\partial z_{r}}\left(\gamma\left[\hat{\gamma} u_{0}+\hat{\varphi} \operatorname{sign}\left(u_{0}\right)\right]+\varphi\right) \\
= & \frac{\partial V_{1}}{\partial z_{1}} z_{2}+\cdots+\frac{\partial V_{1}}{\partial z_{r}} u_{0}+\frac{\partial V_{1}}{\partial z_{r}} \\
& \times\left(-u_{0}+\kappa \gamma u_{0}+\gamma \delta\left\lfloor u_{0}\right\rceil^{2}+\gamma \hat{\varphi} \operatorname{sign}\left(u_{0}\right)+\varphi\right) \\
\leq & \left.-c V_{1}^{\alpha}-\left|\frac{\partial V_{1}}{\partial z_{r}}\right|\left(\kappa \gamma_{m}-1\right)\left|u_{0}\right|+\gamma_{m} \delta\left|u_{0}\right|^{2}+\gamma_{m} \hat{\varphi}-\bar{\varphi}\right) \\
\leq & -c V_{1}^{\alpha}-\gamma_{m}\left|\frac{\partial V_{1}}{\partial z_{r}}\right|(\hat{\varphi}-\bar{\Phi}) \leq-c V_{1}^{\alpha}
\end{aligned}
$$

then, $V_{1}(z)$ converges to zero in finite time, which contradicts the hypothesis. The functions $u_{0}$ and $V_{1}$ are homogeneous, which according to [27], means that $\exists c^{\prime}, \alpha^{\prime}>0:\left|\partial V_{1} / \partial z_{r}\right| \leq$ $c^{\prime} V_{1}{ }^{\alpha^{\prime}}$, where $c^{\prime}=\max _{\left\{z: V_{1}(z)=1\right\}}\left|\partial V_{1} / \partial z_{r}\right|, \quad \alpha^{\prime}=\kappa_{2} / \kappa_{1}$. The terms $\kappa_{2}$ and $\kappa_{1}$ are the respective degrees of homogeneity of $\partial V_{1} / \partial z_{r}$ and $V_{1}$. We suppose now that $V_{1}<\varepsilon$. Let us estimate the overshoot in the worst case condition with respect to uncertainty. For $V_{1}(z(0))=\varepsilon$ and $\hat{\varphi}(0)=0$, we get

$$
\dot{V}_{1} \leq-c V_{1}^{\alpha}-\gamma_{m} c^{\prime} V_{1}^{\alpha^{\prime}}(\hat{\varphi}-\bar{\Phi}), \quad \dot{\hat{\varphi}}=k .
$$

The overshoot $\Delta$ of $V_{1}$ holds for $\dot{V}_{1}=0$ at $t=T_{M}$. We get $\hat{\varphi}\left(T_{M}\right)=\bar{\Phi}-c \Delta^{\alpha-\alpha^{\prime}} / c^{\prime} \gamma_{m} \leq \bar{\Phi}$, and then $T_{M} \leq \bar{\Phi} / k$. An upper bound of $\Delta$ can be estimated as

$$
\Delta=\left(\varepsilon^{1-\alpha^{\prime}}+\frac{c^{\prime}\left(1-\alpha^{\prime}\right) \gamma_{m}}{2 k} \bar{\Phi}^{2}\right)^{\frac{1}{1-\alpha^{\prime}}} .
$$

For an upper bound of $\lim \sup _{t \rightarrow \infty} \hat{\varphi}$, consider the case $V_{1}(z(0))=\varepsilon$ with $\dot{V}_{1}(z(0)) \geq 0$, in this case we have $\hat{\varphi}(0)<\bar{\Phi}$. For $t=T_{M}$, i.e., $\dot{V}_{1}=0$, we get $\hat{\varphi}\left(T_{M}\right) \leq$ $\bar{\Phi}+\hat{\varphi}(0) \leq 2 \bar{\Phi} . \hat{\varphi}$ will increase until time $T_{f}$ where $\dot{\hat{\varphi}}\left(T_{f}\right)=0$ and $V_{1}\left(z\left(T_{f}\right)\right) \geq 0$. The worst case is calculated with respect to the boundary of $\hat{\varphi}$, using $\dot{V}_{1} \leq-c V_{1}^{\alpha}$ and $\dot{\hat{\varphi}}=k$. Here, $T_{f}$ corresponds to $V_{1}\left(z\left(T_{f}\right)\right)=0$, i.e., $T_{f} T_{m}=\left(\Delta^{(1-\alpha)}\right) /(c(1-\alpha))$, which implies that $\hat{\varphi}\left(T_{f}\right) \leq \hat{\varphi}\left(T_{M}\right)+k\left(T_{f} T_{M}\right)=2 \bar{\Phi}+k \Delta^{(1-\alpha)} / c(1-\alpha)$.

Remark 1: The second inequality of 1) of Theorem 2 is equivalent to Levant's concept of real HOSM [28], defined as

$$
\exists t_{1}>0: \forall t>t_{1},\left|z_{i}(t)\right| \leq \mu_{i}, \quad i=1, \ldots, r-1
$$

where $\mu_{i}$ is an arbitrarily small positive number. This is equivalent to practical stability of $z_{1}, \ldots, z_{r}$.

\section{Design AND ImPlementation}

Let us now turn toward design of the robust and adaptive controllers presented in Section III, for PEMFC air-feed system application. As mentioned previously, third-order controllers will be designed in order to obtain a smooth current profile. The test bench is described first in order to present a physical outlook of the system under consideration. Then, the designed controllers and experimental results are presented.

\section{A. Test Bench Description}

The experiments have been conducted on a HIL test bench, presented in Figs. 2 and 3. This bench consists of a twinscrew compressor air-feed system coupled with a real time 


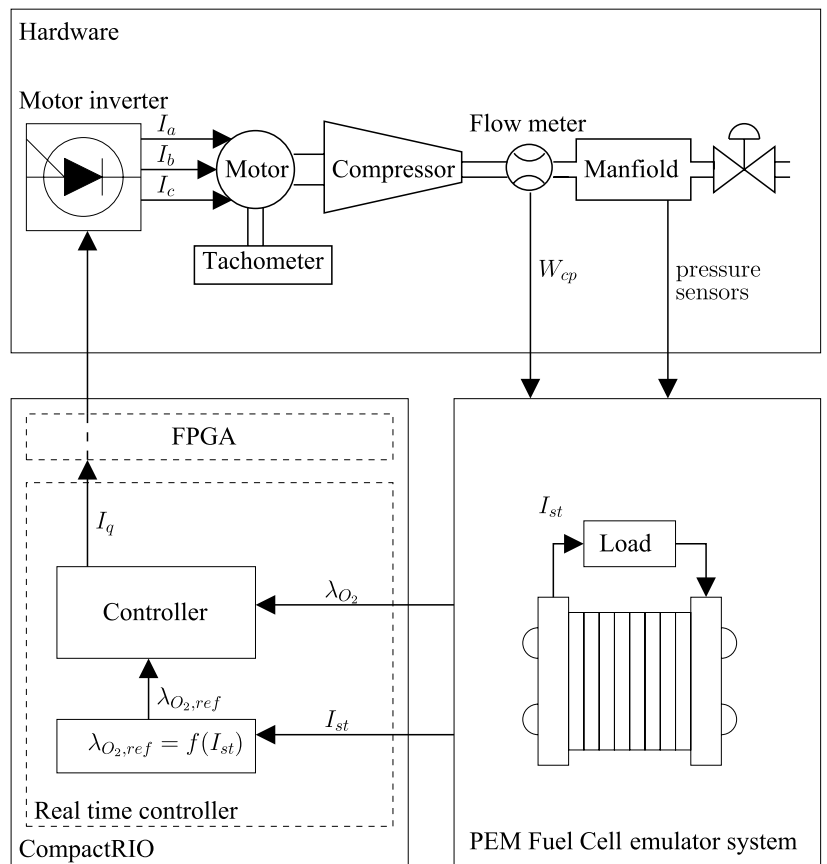

Fig. 3. HIL simulator.

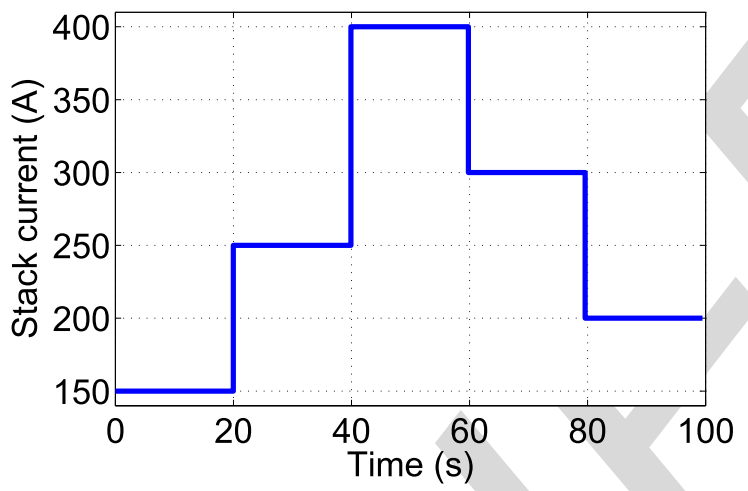

Fig. 4. Stack current (A).

$33-\mathrm{kW}$ fuel cell emulator. The twin-screw compressor is driven by a permanent magnet synchronous motor (PMSM). The three-phase currents of PMSM are calculated from $d q$ coordinates and supplied by an inverter. The control input $I q$ is generated by the proposed controllers installed in a real-time controller and fed to the inverter. The measured compressor airflow $W_{\mathrm{cp}}$ is fed to the real-time fuel cell emulation system. The PEMFC emulator receives the flow rate $W_{\mathrm{cp}}$, in order to generate the states $x_{1}, x_{2}, x_{4}$, and $\lambda_{\mathrm{O}_{2}}$ (see Section II for physical description of the states). Its physical parameters can be varied to emulate the effect of external operating conditions on the fuel cell. The output load is simulated as variable stack current steps between 150 and $400 \mathrm{~A}$, in steps. The load profile used in the tests for this brief is shown in Fig. 4.

\section{B. Controller Design and Experimental Results}

Let us consider (1) and the equations of $\lambda_{\mathrm{O}_{2}}$ and $\lambda_{\mathrm{O}_{2} \text {,ref }}$, i.e., (3) and (4). The system parameters are given in [22]. The sliding variable is defined as $z_{1}=s=\lambda_{\mathrm{O}_{2}}-\lambda_{\mathrm{O}_{2}}$,ref.
In our case, the sliding variable $s$ depends on $x_{1}, x_{2}$, and $x_{4}$. The first and second time derivative of $s$ are

$$
\begin{aligned}
\dot{s}=z_{2}= & \frac{\partial}{\partial x_{1}} s\left(x_{1}, x_{2}, x_{4}\right) \cdot \dot{x}_{1}\left(x_{1}, x_{2}, x_{4}\right) \\
& +\frac{\partial}{\partial x_{2}} s\left(x_{1}, x_{2}, x_{4}\right) \cdot \dot{x}_{2}\left(x_{1}, x_{2}, x_{4}\right) \\
& +\frac{\partial}{\partial x_{4}} s\left(x_{1}, x_{2}, x_{4}\right) \cdot \dot{x}_{4}\left(x_{1}, x_{2}, x_{3}, x_{4}\right) \\
\ddot{s}=z_{3}= & \frac{\partial}{\partial x_{1}} \dot{s}\left(x_{1}, x_{2}, x_{3}, x_{4}\right) \cdot \dot{x}_{1}\left(x_{1}, x_{2}, x_{4}\right) \\
& +\frac{\partial}{\partial x_{2}} \dot{s}\left(x_{1}, x_{2}, x_{4}\right) \cdot \dot{x}_{2}\left(x_{1}, x_{2}, x_{4}\right) \\
& +\frac{\partial}{\partial x_{3}} \dot{s}\left(x_{1}, x_{2}, x_{3}, x_{4}\right) \cdot \dot{x}_{3}\left(x_{3}, x_{4}, u\right) \\
& +\frac{\partial}{\partial x_{4}} \dot{s}\left(x_{1}, x_{2}, x_{3}, x_{4}\right) \cdot \dot{x}_{4}\left(x_{1}, x_{2}, x_{3}, x_{4}\right) .
\end{aligned}
$$

333

The control input $u$ appears for the first time in the second time derivative of $s$. To obtain a continuous control $u$, the discontinuous control is applied on the higher derivative $\dot{u}$. We get

$$
s^{(3)}=\dot{z}_{3}=\underbrace{\dot{\varphi}+\dot{\gamma} u}_{\Phi}+\gamma v
$$

where $v=\dot{u}$, $\Phi$, and $\gamma$ are uncertain bounded functions that satisfy

$$
\Phi \in[-\bar{\varphi}, \bar{\varphi}], \quad \gamma \in\left[\gamma_{m}, \gamma_{M}\right] .
$$

For the PEMFC under consideration, the bounding values of the parameters were determined as percentage deviations through precise physical analyses. The numerical values of the uncertainty limits were obtained as $\bar{\varphi}=0.03, \gamma_{m}=5$, and $\gamma_{M}=15$.

From here, the control objective becomes equivalent to forcing $s$, and its first and second time derivatives to zero in finite time, through $s^{(3)} \in[-\bar{\varphi}, \bar{\varphi}]+\left[\gamma_{m}, \gamma_{M}\right] v$. We first develop a third-order SMC robust controller using (11) and (12). According to Theorem 1, the controller takes the following structure:

$$
\begin{aligned}
v_{1} & =-l_{1}\lfloor s\rceil^{\alpha_{1}} \\
v_{2} & =-l_{2}\left\lfloor\lfloor\dot{s}\rceil^{\beta_{1}}-\left\lfloor v_{1}\right\rceil^{\beta_{1}}\right\rceil^{\alpha_{2} / \beta_{1}} \\
v_{3} & =-l_{3}\left\lfloor\lfloor\dot{s}\rceil^{\beta_{2}}-\left\lfloor v_{2}\right\rceil^{\beta_{2}}\right\rceil^{\alpha_{3} / \beta_{2}} \\
v & =\dot{u}=\frac{1}{\gamma_{m}}\left(v_{3}+\bar{\varphi} \operatorname{sign}\left(v_{3}\right)\right) .
\end{aligned}
$$

In this test, the parameters have been tuned to the following values: $l_{1}=5, l_{2}=10, l_{3}=40, \beta_{0}=0.8, \beta_{1}=1.25$, $\beta_{2}=2, \alpha_{1}=4 / 5, \alpha_{2}=3 / 4, \alpha_{3}=2 / 3, \gamma_{m}=5, \bar{\varphi}=0.03$.

The load variations (Fig. 4) result in changes in $\lambda_{\mathrm{O}_{2} \text {,ref }}$, according to (4). The performance of the robust controller with respect to these changes is shown in Fig. 5. It can be seen in Fig. 5(a) that $\lambda_{\mathrm{O}_{2}}$ tracks $\lambda_{\mathrm{O}_{2, \text { ref }}}$ successfully with a response time between 3 and $7 \mathrm{~s}$ practically. The control input $\left(I_{q}\right)$ is shown in Fig. 5(b), it varies between 0 and $3 \mathrm{~A}$. As the controller establishes third-order HOSM, the oscillations in $I_{q}$ are negligible and it has a smooth profile. 


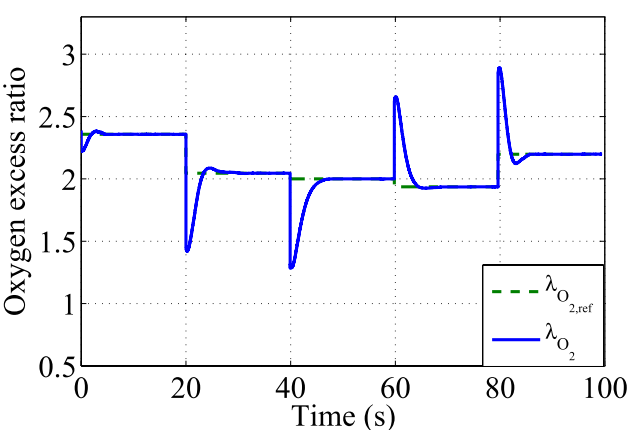

(a)

Fig. 5. Robust controller. (a) $\lambda_{\mathrm{O}_{2}}$. (b) $I_{q}$.

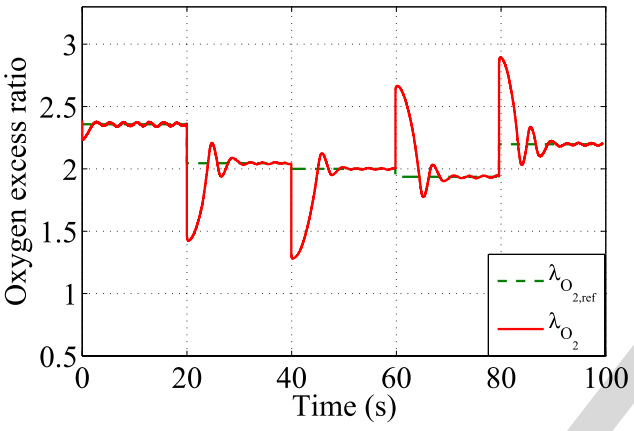

(a)

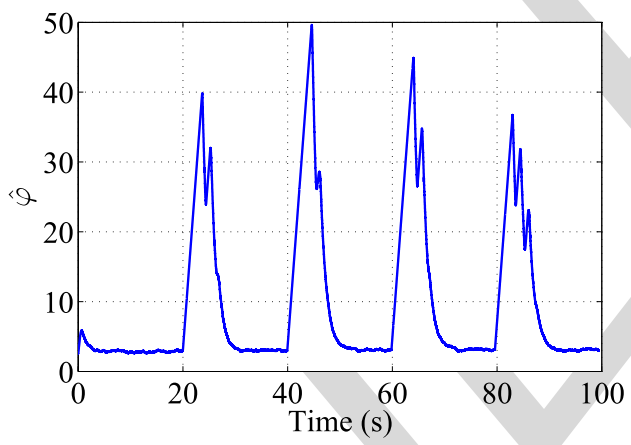

(c)

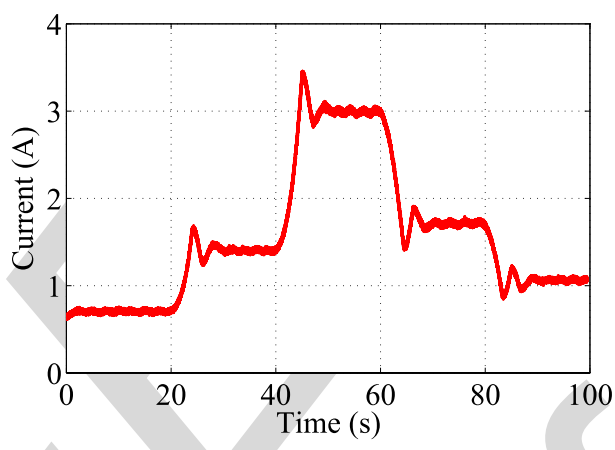

(b)

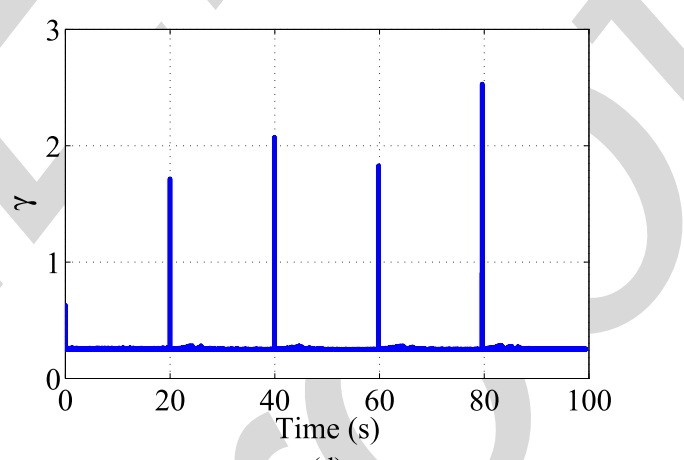

(d)
Fig. 6. Adaptive controller. (a) $\lambda_{\mathrm{O}_{2}}$. (b) $I_{q}$. (c) $\hat{\varphi}$. (d) $\hat{\gamma}$.
We will now demonstrate the proposed adaptive controller for the same problem, assuming that we have no knowledge of the uncertainty bounds. The third-order SMC adaptive controller is designed using (12) and (13). According to Theorem 3, the controller has the following structure:

$$
v=\dot{u}=\hat{\gamma} v_{3}+\hat{\varphi} \operatorname{sign}\left(v_{3}\right)
$$

where $v_{3}$ is the same as in (17). The controller parameters used in adaptive case are as follows: $l_{1}=5$, $\alpha_{1}=4 / 5, \alpha_{2}=3 / 4, \alpha_{3}=2 / 3, k=5, \eta=0.95$, $\varepsilon=0.001, \kappa=0.25, \delta=0.001$.

The results of the adaptive controller are shown in Fig. 6. Fig. 6(a) shows that $\lambda_{\mathrm{O}_{2}}$ converges and remains inside a small and acceptable neighborhood around the desired value $\lambda_{\mathrm{O}_{2} \text {,ref. }}$. The control input, $I_{q}$ is shown in Fig. 6(b) and the behaviors of the adaptive parameters $\hat{\varphi}$ and $\hat{\gamma}$ are shown in Fig. 6(c) and (d), $l_{2}=10, \quad l_{3}=40, \quad \beta_{0}=0.8, \quad \beta_{1}=1.25, \quad \beta_{2}=2$,

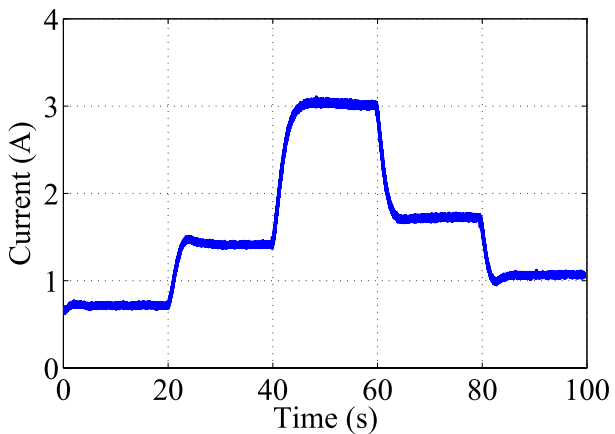

(b) respectively. It can be seen that $\hat{\varphi}$ increases at each stack current step, and then decreases rapidly after the convergence of the tracking error. As ideal sliding mode cannot be achieved in this case, small oscillations can be seen in $\hat{\gamma}$. In general, these results show the effectiveness of both the robust and adaptive controllers for a wide range of stack current variation, i.e., external perturbation.

To demonstrate the robustness of our controllers in dealing with parametric uncertainty, another series of experiments was conducted, in which the parameters of the PEMFC emulator were varied to their extreme values [22]. The designed controllers were again tested with the same controller parameters as determined before. The results of the robust controller in these tests are shown in Fig. 7. The system response in Fig. 7(a) and the control input in Fig. 7(b) show that this controller performs as well as in the previous tests with defined system parameter values. The results of 


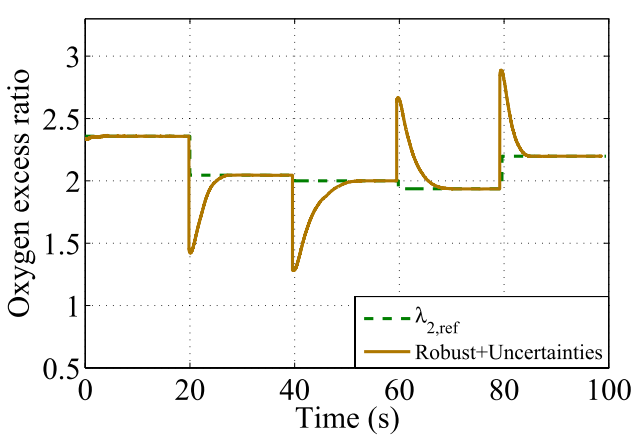

(a)

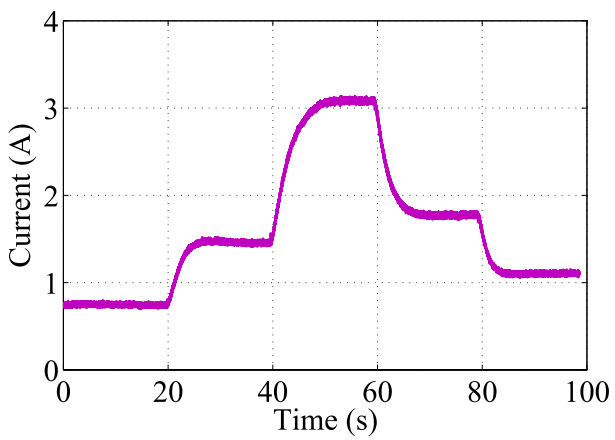

(b)

Fig. 7. Robust controller (parametric shift). (a) $\lambda_{\mathrm{O}_{2}}$. (b) $I_{q}$.

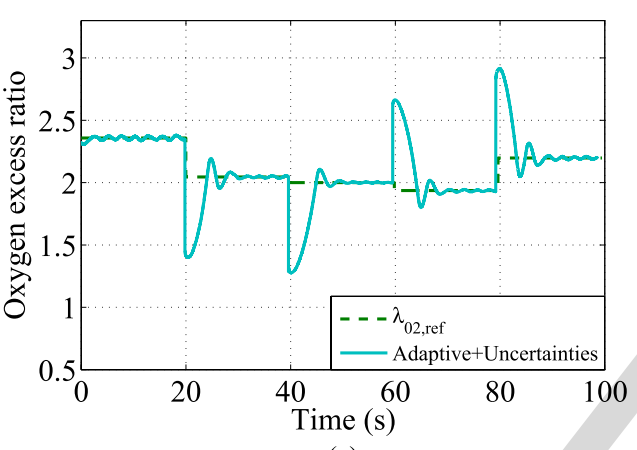

(a)

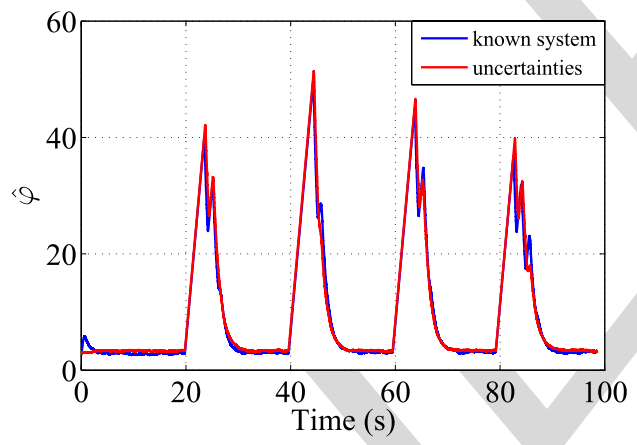

(c)

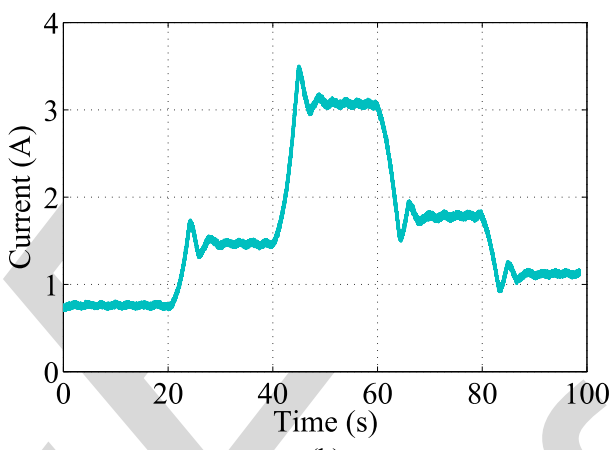

(b)

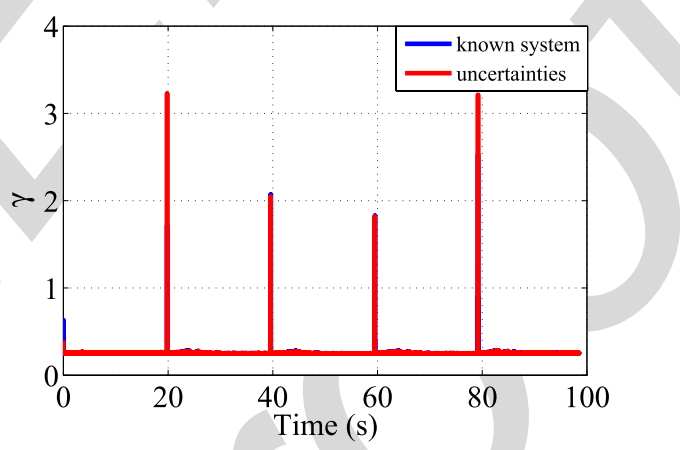

(d)

Fig. 8. Adaptive controller (parametric shift). (a) $\lambda_{\mathrm{O}_{2}}$. (b) $I_{q}$. (c) $\hat{\varphi}$. (d) $\hat{\gamma}$.

the adaptive controller are shown in Fig. 8. We see again in Fig. 8(a) a similar behavior of tracking error as compared with the previous tests. However, the static value of the quadratic current [Fig. 8(b)] changes in order to accommodate the emulated parametric drift. The adaptive gains $\hat{\varphi}$ and $\hat{\gamma}$ adapt to counteract the uncertainty, ensuring convergence, as seen in Fig. 8(c) and (d). These tests demonstrate that both the robust and adaptive controllers are capable of handling parametric uncertainty, albeit with different mechanisms.

\section{CONCLUSION}

In this brief, the control problem of PEMFC air-feed system was addressed using two arbitrary HOSM controllers associated with the finite-time stabilization of a perturbed chain of integrators with bounded uncertainty. The design of the first controller requires the knowledge of the uncertainty bounds. The second controller is adaptive and its design does not require any quantitative knowledge of the uncertainty bounds.
The arbitrary order nature of controllers permitted to extend the air-feed system from second to third order, resulting in continuous input current profile. The proposed controllers showed good performance in simulation and in experiments conducted on a PEMFC air-feed test bench.

\section{REFERENCES}

[1] J. T. Pukrushpan, A. G. Stefanopoulou, and H. Peng, Control of Fuel Cell Power Systems: Principles, Modeling, Analysis and Feedback Design. New York, NY, USA: Springer-Verlag, 2004.

[2] A. Y. Karnik, A. G. Stefanopoulou, and J. Sun, "Water equilibria and management using a two-volume model of a polymer electrolyte fuel cell," J. Power Sour., vol. 164, no. 2, pp. 590-605, 2007.

[3] A. Vahidi, I. Kolmanovsky, and A. Stefanopoulou, "Constraint handling in a fuel cell system: A fast reference governor approach," IEEE Trans. Control Syst. Technol., vol. 15, no. 1, pp. 86-98, Jan. 2007.

[4] S. V. Emel'yanov, S. K. Korovin, and A. Levant, "High-order sliding modes in control systems," Comput. Math. Model., vol. 7, no. 3, pp. 294-318, 1996.

[5] V. I. Utkin, Sliding Modes in Control and Optimization. Berlin, Germany: Springer-Verlag, 1992.
427 428 429 430 431 
[6] A. Levant, "Universal single-input-single-output (SISO) sliding-mode controllers with finite-time convergence," IEEE Trans. Autom. Control, vol. 46, no. 9, pp. 1447-1451, Sep. 2001.

[7] A. Levant, "Homogeneity approach to high-order sliding mode design," Automatica, vol. 41, no. 5, pp. 823-830, 2005.

[8] M. Defoort, T. Floquet, A. Kokosy, and W. Perriquetti, "A novel higher order sliding mode control scheme," Syst. Control Lett., vol. 58, no. 2, pp. 102-108, 2009.

[9] F. Dinuzzo and A. Fererra, "Higher order sliding mode controllers with optimal reaching," IEEE Trans. Autom. Control, vol. 54, no. 9, pp. 2126-2136, Sep. 2009.

[10] Y.-J. Huang, T.-C. Kuo, and S.-H. Chang, "Adaptive sliding-mode control for nonlinearsystems with uncertain parameters," IEEE Trans. Syst., Man, Cybern. B, Cybern., vol. 38, no. 2, pp. 534-539, Apr. 2008.

[11] F. Plestan, Y. Shtessel, V. Brégeault, and A. Poznyak, "New methodologies for adaptive sliding mode control," Int. J. Control, vol. 83, no. 9 , pp. 1907-1919, 2010

12] F. Plestan, Y. Shtessel, V. Brégeault, and A. Poznyak, "Sliding mode control with gain adaptation-Application to an electropneumatic actuator," Control Eng. Pract., vol. 21, no. 5, pp. 679-688, 2013.

[13] Y. Shtessel, M. Taleb, and F. Plestan, "A novel adaptive-gain supertwisting sliding mode controller: Methodology and application," Automatica, vol. 48, no. 5, pp. 759-769, 2011.

[14] A. Dávila, J. A. Moreno, and L. Fridman, "Variable gains super-twisting algorithm: A Lyapunov based design," in Proc. Amer. Control Conf., Baltimore, MD, USA, Jun./Jul. 2010, pp. 968-973.

[15] M. Taleb, A. Levant, and F. Plestan, "Pneumatic actuator control: Solution based on adaptive twisting and experimentation," Control Eng. Pract., vol. 21, no. 5, pp. 727-736, 2013.

[16] A. Glumineau, Y. B. Shtessel, and F. Plestan, "Impulsive-sliding mode adaptive control of second order system," in Proc. 18th IFAC World Congr., Milan, Italy, Aug./Sep. 2011, pp. 5389-5394.

[17] M. Harmouche, S. Laghrouche, and Y. Chitour, "Robust and adaptive higher order sliding mode controllers," in Proc. 51st IEEE Conf. Decision, Control, Eur. Control Conf., Orlando, FL, USA, Dec. 2012 pp. 6436-6441.
[18] W. Gracia-Gabin, F. Dorado, and C. Bordons, "Real-time implementation of a sliding mode controller for air supply on a PEM fuel cell," J. Process Control, vol. 20, no. 3, pp. 325-336, 2010.

[19] C. Kunusch, P. F. Puleston, M. A. Mayosky, and J. Riera, "Sliding mode strategy for PEM fuel cells stacks breathing control using a supertwisting algorithm," IEEE Trans. Control Syst. Technol., vol. 17, no. 1, pp. 167-174, Jan. 2009.

[20] I. Matraji, S. Laghrouche, and M. Wack, "Cascade control of the motocompressor of a PEM fuel cell via second order sliding mode," in Proc 50th IEEE Conf. Decision, Control, Eur. Control Conf. (CDC-ECC), Dec. 2011, pp. 633-638.

[21] C. Kunusch, P. F. Puleston, M. Á. Mayosky, and L. Fridman, "Experimental results applying second order sliding mode control to a PEM fuel cell based system," Control Eng. Pract., vol. 21, no. 5, pp. 719-726, 2013.

[22] I. Matraji, S. Laghrouche, S. Jemei, and M. Wack, "Robust control of the PEM fuel cell air-feed system via sub-optimal second order sliding mode," Appl. Energy, vol. 104, pp. 945-957, Apr. 2013.

[23] A. F. Filippov, Differential Equations with Discontinuous Righthand Sides. Dordrecht, The Netherlands: Kluwer, 1988.

[24] S. P. Bhat and D. S. Bernstein, "Finite-time stability of continuous autonomous systems," SIAM J. Control Optim., vol. 38, no. 3 , pp. 751-766, 2000.

[25] Y. Hong, "Finite-time stabilization and stabilizability of a class of controllable systems," Syst. Control Lett., vol. 46, no. 4, pp. 231-236, 2002.

[26] X. Huang, W. Lin, and B. Yang, "Global finite-time stabilization of a class of uncertain nonlinear systems," Automatica, vol. 41, no. 5, pp. 881-888, 2005.

[27] S. P. Bhat and D. S. Bernstein, "Geometric homogeneity with applications to finite-time stability," Math. Control, Signals, Syst., vol. 17, no. 2, pp. 101-127, 2005.

[28] A. Levant, "Sliding order and sliding accuracy in sliding mode control," Int. J. Control, vol. 58, no. 6, pp. 1247-1263, 1993. 